price to pay for the simplicity of basing all calculations on the simple sum of two Index numbers, one for the port and one for the day's tidal range.

The base of the logarithms, which determines the increment in half range between one column and the next, has been arbitrarily chosen to give reasonable steps. The Tide Index varies between $\circ$, for a tide less than mean neaps, and I 8 for extreme springs. For the coast between Brest and the Gironde the Port Index varies between o and 5 .

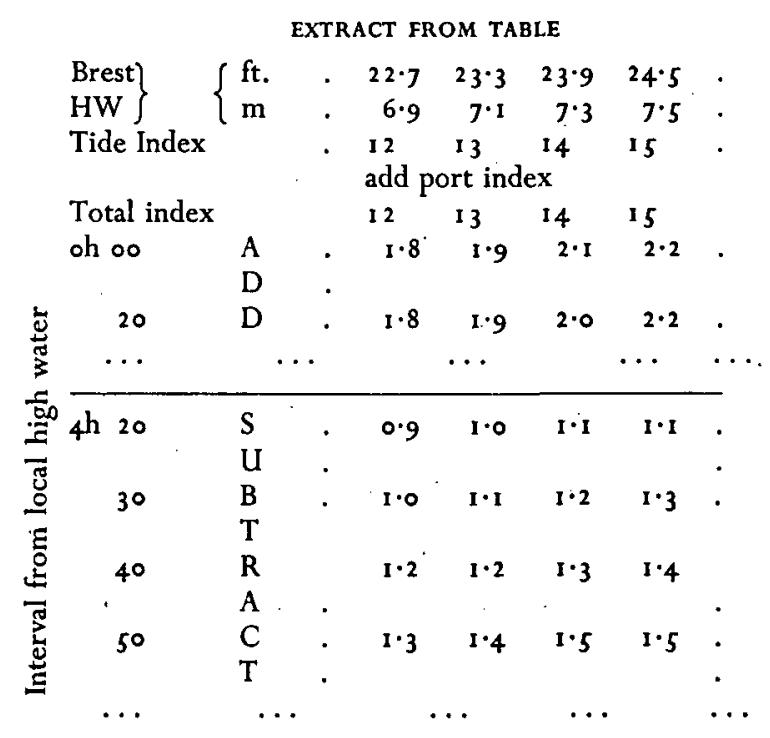

\title{
Revised Rules for Preventing Collisions at Sea
}

\section{Rear-Admiral J. García-Frías}

Captain P. A. Thompson's paper, Revised Rules for Preventing Collisions at Sea, in the January issue of the Journal should be discussed. I agree with him that 'rules (the ones which are mainly based on the operational aspect ${ }^{2}$ of the collision problem, i.e. manœuvring rules) to cover every conceivable circumstance would be of such length as to be impracticable.' However, in my opinion, rules based on the principles-the organic aspects ${ }^{2}$ - can cope practically because then the object of the rules is to define the collision problem and its solution so that use of the rules can be immediate, easy and effective. For example, by establishing a safety distance ${ }^{1}$ the passing operation would have to be carried out such that the miss distance would always be greater than the safety distance. Furthermore, by keeping the ship's range above the safety distance, one ensures 
that the entire operation is correctly carried out. Finally, should the range become less than the safety distance, it is a good indication-as long as own ship has manœuvred correctly-that the other vessel has acted incorrectly. She may have failed to manœuvre at all or may have done so incorrectly, but whatever the reason, with her failure so revealed it is possible to adopt precautionary measures. Thus, the essential thing in constructing the rules is to establish the conditions for action in a dangerous situation so that when either general or specific problems arise decisive action may be taken. An analysis of these problems will show the principles which must be established so that action would always be easy and effective.

The problem clearly shows that rules, based on action only-for instance, those proposed by Captain Thompson-cannot properly solve the collision problem. This is because if, as he maintains, the same action may be applied in both clear weather and in restricted visibility, the same thing cannot be said about the general principles governing all the questions inherent ${ }^{2}$ in the collision problem as these are very different in each situation. This is so because the two elements defining the situation in an encounter-bearing and range-are presented in very different ways. In visual presentation, bearing has a greater weight since bearing from the other's aspect is only available in this situation and own bearing has the same weight as it would in a radar situation. On the other hand, range is very roughly estimated visually, though radar gives it accurately enough. This is why rules must be different in each situation though the principles must be the same to give a basic uniformity, simplicity, so that it would be possible to handle a change in visibility conditions easily. In other words, the fundamental principles must be the same, though their implementation must differ. ${ }^{3}$

The problem of responsibility is solved by Captain Thompson in various ways: dual responsibility for conventional vessels each having the other forward of her beam; single responsibility for a conventional vessel with a hampered vessel forward of her beam and for sailing vessels between them; no responsibility at all for hampered vessels in general or for conventional vessels having another vessel abaft her beam. This last idea-that of no responsibility at all for vessels having another vessel abaft her beam is a new and very original idea in the collision problem, and it is interesting to note that in the Steering and Sailing Rules, when one vessel is compelled to keep out of the way of another, the latter must keep a steady course and speed. Besides these different sorts of responsibility, Captain Thompson proposes dual responsibility for any of the previously mentioned vessels if they 'find themselves so close that collision cannot be avoided by following (her) manœuvres.' Thus, Captain Thompson's proposal fails in the same way as the much-discussed Rule 21 . My proposals ${ }^{3}$ make dual responsibility universal-but in a relative way. The capability of the vessels and the circumstances of the case would be taken into account so that sometimes the situation might work out as it would have in cases of single responsibility. However, this solution of universal responsibility is only possible with the guarantee of safety implied in my Sector Rule.

Obviously, as is commonly said, all roads lead to Rome. Nevertheless port-toport passings are the most frequent in practice and some of these encounters may only require a slight turn to port to allow a safe pass, while turning to starboard would consume much time and sea-room. The reason for Captain Thompson adopting the approach establishing that 'any action, in avoiding another vessel must be performed with the object of making the line-of-sight of the other 
vessel rotate in an anti-clockwise direction' seems principally designed to avoid the problem of ambiguity which may arise because of differing interpretations of radar information leading to disagreement, on both vessels, about the correct application of the manouvring rules.

This ambiguity - though it must be said that this is not an essential problem as it can be solved by the application of the Sector Rule 2 -may be easily solved by the step-by-step manœuvre in a way covering any kind of vessel using radar in a reduced visibility situation. This method of solution is proposed by the author in another contribution in the Forum section of this Journal. In the case of good visibility the criterion could be that vessel $A$ with the other $B$ on her starboard must initiate action in accord with the manouvring rules. And that vessel B must take action as soon as she knows what vessel $A$ is doing. So, the case could arise, if vessel A is hampered, where her conduct would be contrary to the general manouvring rules. If this should occur vessel B must, and would be able to, adjust her actions to the situation. On the other hand, Captain Thompson's Steering Rules cannot cover all the situations involving sailing vessels, and he must give them a special rule (Rule 4) which may contradict his Rule I 6.

The idea of increasing speed, as found in Rule 2(c), seems an unwise solution to be specified in the Rules because it is well known that, in general, the reserve over normal speed is small. The agreement established in Rule $2(\mathrm{e})$ also seems unwise.

Finally, it seems that Captain Thompson, with his Rule 5(c), intends to cope with the problem of two or more vessels simply by establishing that: 'At the junction of routes, vessels shall proceed round them in an anti-clockwise direction.' In fact this is the direction implied in my tactics for vessel group manœuvres, 2 but in order to arrange this multi-vessel situation safely, it is necessary to establish the limits of the range at which a vessel becomes a part of a group, the classification of peripheral and internal vessels, and the Sector Rule, as essential to common safety while using radar in reduced visibility.

\section{REFERENCES}

1 García-Frías, J. (1960). Anti-collision radar sectors. This Journal, 13, 316.

2 Garcia-Frias, J. (1965). The sector rules and the collision problem. This Journal, 18, 141 .

3 Garcia-Frías, J. (1970). The revision of the Rules. This Journal, 23,7 I.

\section{The Collision Problem and Manœuvring by Stages}

\section{Rear-Admiral J. García-Frías}

WHEN the danger of collision between vessels occurs, the proper interpretation of radar information will indicate that, with respect to the manouvring rules, any avoiding action should always be positive. However, it is well known that radar 